\title{
Agencia moral corporativa Un concepto integrador
}

\author{
Martha M. Rodríguez Coronel ${ }^{1}$ \\ al228496@uji.es
}

\footnotetext{
${ }^{1}$ Agradecimientos al Banco Santander por su apoyo en mi desarrollo académico a través de sus becas de máster y doctorado.
} 
El objetivo del presente artículo es crear un concepto integrador de agencia moral corporativa, a partir de la analogía con la agencia moral individual, vinculado con tres propuestas colectivistas que serán examinadas y constituirán la base de nuestro concepto. La elaboración de este concepto integrador de agencia moral responde a una doble finalidad ulterior: por un lado, fundamentar el deber de las empresas de responder ante la comunidad moral, y con ello facilitar para futuras investigaciones la construcción de una noción de engagement ético. La metodología utilizada es de naturaleza teórica-cognoscitiva, desarrollada argumentativamente. En la primera parte se analizan las tres tesis colectivistas fundamentales de la agencia moral corporativa. En la segunda sección se exponen las replicas a dichas tesis y algunos puntos en contra de la responsabilidad de la empresas. En la tercera parte se ofrece una propuesta propia de agencia moral corporativa. Por último se hace una revisión de todos los puntos mencionados presentada a manera de conclusiones.

Palabras clave: ética empresarial, agente moral, comunidad moral.

\section{Introducción}

La ética empresarial es la ética aplicada que estudia los problemas éticos que surgen en el seno de las corporaciones. Esta rama de la ética evalúa el comportamiento individual y el comportamiento corporativo en las situaciones en las que las empresas deban o no comprometerse. Asimismo "describe y evalúa la gerencia corporativa, la comunidad corporativa y las interacciones de la empresa con el medio ambiente» (Freeman y Werhane, 2009: 517-518).

Uno de los principales focos de estudio de la ética empresarial es la naturaleza de la corporación, que procura, entre otros aspectos, responder la pregunta sobre la capacidad de las empresas de ser responsables moralmente, como lo es una persona. En los últimos años, este tema ha cobrado mayor importancia en la comunidad corporativa mundial, porque ha habido algunos escándalos y sospechas sobre la confiabilidad y transparencia del funcionamiento de las organizaciones, y más aún sobre las consecuencias de sus actos. La sociedad responsabiliza a las empresas de sus errores y de sus pésimas políticas laborales o medioambientales. Sin embargo, a quién se está culpando realmente, a los altos ejecutivos como individuos o a la corporación como si fuera un sujeto.

La conceptualización de la responsabilidad social corporativa ha estado implícita en los planes que desarrollan las empresas, ya sea a nivel local, regional o global. Parece que tácitamente se está atribuyendo 
responsabilidad a las empresas. Pero no está claro en qué sentido esa responsabilidad es diferente, o la misma, de la responsabilidad de las personas que conforman, dirigen o poseen la organización. Por ello es tan importante esclarecer si la empresa como tal es un agente moral, pues con ello tendrán que ver otras cuestiones muy relevantes, como por ejemplo, si tienen o no derechos, como el derecho a la propiedad, o deberes, y entre tales deberes el principal puede ser el de colaborar activa y sinceramente en la sociedad.

Las empresas son más que simples máquinas, pero para adquirir el estatus de agentes morales tendrían que poseer razones, suyas y no sólo de sus miembros, que justifiquen lo que se hace en dichas corporaciones. Claro está que las corporaciones no pueden pensar por sí solas, pero en la medida en que su nombre aparece como garante de justificaciones, y en la medida, además, que se pueden interponer demandas o quejas a las mismas, y no sólo a sus miembros, y que eso hace que se les atribuya responsabilidad en algún sentido, sí parecen poseer la estructura apropiada para ser responsables. Así, según Thomas Donaldson, pudieran serlo y dar una explicación moral a las causas que provocan su comportamiento (1982). También podrían tener la capacidad de controlar sus políticas organizacionales, tratando de promover determinados límites morales a través de reglas y procedimientos.

Para calificar a una corporación como agente moral, ésta necesitaría poder personificar de alguna manera las características del agente moral individual, adaptándolas al sistema organizacional para lograr un proceso moral de toma de decisiones. En principio, pareciera que si una compañía tiene la capacidad de utilizar razones morales en la toma de decisiones y la facultad de controlar, no sólo los actos corporativos sino también la estructura de políticas y reglas en el proceso de toma de decisiones, entonces poseería el estatus de agente moral, pero la cuestión es más compleja. No parece que baste tan sólo con cumplir estas condiciones, ya que el problema fundamental planteado a partir de aquí sería de quién son esas razones morales empresariales y dónde reside el origen de su responsabilidad y de sus reglas: si en la empresa como sujeto suprapersonal -diferente en algún sentido a sus miembros- o meramente en los individuos que representan a esa empresa.

Pero realmente ¿qué significa considerar a las organizaciones como agentes morales? ¿las organizaciones compartirían todos los derechos y obligaciones naturales de los humanos? ¿se ajustarían a las reglas de la comunidad moral y se auto-regularían a través del razonamiento moral? Como respuesta a estas preguntas han surgido posiciones a favor, y en contra de la agencia moral corporativa. 


\section{Objetivos}

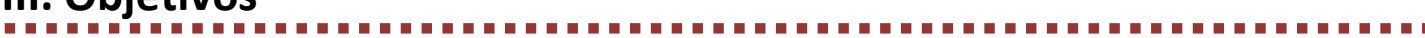

- Desarrollar las tesis corporativistas de Peter French, de Kenneth Goodpaster y John Mathews, y de Adela Cortina.

- Explorar la réplica individualista realizada por Nani Ranken, y las posiciones de otros detractores.

- Proponer un concepto integrador de agencia moral corporativa.

\section{Propuestas colectivistas}

\section{Peter French}

A lo largo de la historia solamente se ha encontrado un concepto de persona jurídica que está vinculado a un sentido metafísico, este concepto es el de la definición clásica medieval "Una persona es una substancia individual de naturaleza racional» (Boecio en Marebon, 2003: 71), en otras palabras, es un sujeto dotado de razón por naturaleza. El resto de los conceptos de persona jurídica son prácticamente inútiles para cualquier propósito moral en la búsqueda de situar a las empresas como agentes morales (French, 1979: 208). Luego de examinar la teoría de la ficción $^{2}$ y de la agregación ${ }^{3}$, Peter French deduce que la existencia biológica no está asociada esencialmente al concepto de persona y que el paradigma de una definición neutral inclusiva de la persona moral es proporcionada por un sujeto de derecho, definiendo así a la persona moral como un sujeto con responsabilidad atribuida de segundo tipo (1979: 210). A continuación, se desarrollará la teoría del autor a partir de este concepto de agente moral, particularmente aplicado a las empresas.

La propuesta de agencia moral corporativa de P. French está centrada en la intencionalidad (González, 2001: 322). El núcleo explicativo de esta postura reside en el intento de proporcionar la justificación de una teoría que permita tratar a las empresas como miembros de la comunidad moral, con el mismo estatus que los seres humanos que ya han sido

\footnotetext{
${ }^{2}$ La definición tradicional de persona en la antigua Roma establecía que las personas eran creaciones de la ley, ignorando cualquier consideración biológica o metafísica. A partir de esa noción se desarrolla la teoría de la ficción. Según ésta, la personalidad de una organización es una invención y debe su existencia a un mero acto creativo del estado. Solamente reconoce aquellos sujetos adscritos a su marco normativo (Pettet, 2005: 48; French, 1979: 208).

${ }^{3}$ La teoría de la agregación no posee una única definición, pero en casi todas se encuentra presente la idea de las organizaciones como varios grupos cohesionados que poseen unidades más pequeñas, las personas. En palabras de Robert Hessen, la teoría de la agregación sostiene que cualquier tipo de organización, sin importar su estatus legal, es simplemente una asociación de individuos que actúan unidos para alcanzar una meta en común. La membrecía de una organización se mantiene para sumar agregados, pero la organización no es la suma de los derechos y deberes de sus miembros. Entonces, una entidad inexistente no puede actuar, ni formar intenciones, ni tener conocimientos o razones para conocer, ni tener responsabilidad moral (Hessen, 1979: 41; Freund, 2000: 53).
} 
reconocidos tradicionalmente como tales. En palabras de P. French: «las empresas pueden ser personas morales auténticas y tener todo tipo de privilegios, derechos y deberes como los que se conceden a las personas morales en el curso normal de acontecimientos» (French, 1979: 207).

Para que una empresa sea tratada como un agente ${ }^{4}$ se debe poder describir o re-describir ${ }^{5}$ sus acciones como intencionadas ${ }^{6}$ y que sean realizadas por la propia empresa. P. French sostiene que para hacer esto posible es necesario que la organización posea una estructura de decisión interna (EDI) que le permita mantener y difundir la intencionalidad de la empresa, demostrando que las empresas, y no simplemente sus trabajadores, tienen razones para hacer lo que hacen. Esta estructura posee dos elementos, un organigrama que establece estaciones y niveles dentro de la distribución de la empresa y unas reglas de reconocimiento para tomar decisiones corporativas, es decir, la política empresarial (French, 1979: 212). Las funciones de la EDI son, tanto guiar a todos los niveles de la empresa en el proceso de toma de decisiones y en la ratificación de las mismas, como lograr la subordinación y síntesis de las intenciones y actuaciones de los individuos en el momento de decidir alguna cuestión empresarial. $^{7}$

El organigrama distingue quiénes son los jugadores y aclara su rango y su responsabilidad con respecto a los otros dentro de la organización, es decir, delinea las relaciones interdependientes y dependientes, directas o de grupo, que están involucradas en la determinación de las actuaciones de la empresa. Las reglas de reconocimiento permiten que las decisiones sean fijadas colectivamente en varios niveles, y además sean ratificadas por los niveles más altos de la corporación (French, 1979: 213).

La importancia de esta estructura para la corporación se encuentra en que debe subordinar las decisiones y ambiciones individuales para la supervivencia y bienestar organizacional, por ello debe tener una colección de principios y unas reglas de conductas que limiten y dirijan las actuaciones y los comportamientos de sus miembros. Por otro lado, la EDI hace posible la transformación descriptiva de los eventos que le atribuye la intencionalidad necesaria para ser agentes morales, diferenciando a la corporación de una simple multitud de personas (French, 1979: 215).

\footnotetext{
${ }^{4}$ En términos de agente davidsoniano, «un hombre es un agente de una acción si lo que hace puede ser descrito bajo un aspecto de intencionalidad» (Davidson, 2001: 46).

${ }^{5}$ Véase el ejemplo de Hamlet (Davidson, 2001: 46).

${ }^{6}$ Véase el ejemplo del derramamiento de café (Davidson, 2001: 65).

${ }^{7}$ Cuando los directores votan en una junta acerca del futuro de una empresa en el marco de la EDI, también la corporación está decidiendo hacer algo, sin consideración de las razones personales que los ejecutivos tengan para votar como lo hacen; y aun si esas razones personales fueran inconsistentes con la política organizacional, la corporación obra por sus propias razones y los actos deberían serle atribuidos a ella.
} 


\section{Goodpaster y Mathews}

Kenneth Goodpaster y John Mathews sostienen que las corporaciones no sólo poseen la capacidad de tener conciencia, sino que deben tenerla. También afirman que las empresas son tan responsables como los individuos y que el lenguaje ético tiene un lugar preponderante en el vocabulario organizacional.

Estos autores toman su posición a través de una analogía entre el individuo y las empresas, analizando primeramente cómo funciona el concepto de responsabilidad moral en las personas, para luego extrapolarlo a las organizaciones y conseguir caracterizarlas también como agentes morales. Su objetivo no es ofrecer una guía o estructura para la validación de las decisiones de las empresas, que sirva para armar o constituir su responsabilidad moral -como P. French lo hizo a través de la EDI, sino el de aclarar los cimientos conceptuales de la ética empresarial, específicamente en la atribución de las empresas como agente morales (Goodpaster y Mathews, 1982: 3-6).

Para comenzar, los autores explican las tres acepciones de responsabilidad concernientes a las personas como individuos. El primer significado que exponen es el de responsabilidad causal, ${ }^{8}$ luego el de la responsabilidad del seguimiento de reglas, ${ }^{9}$ y por último, se encuentra la responsabilidad en la toma de decisiones en la que los autores se enfocan. Para que un individuo sea considerado como responsable debe ser confiable y mantener una actitud controlada y racional al momento de actuar para decidir. En esta acepción es que reside la característica distintiva de la responsabilidad moral, que «subraya la intencionalidad y la independencia del juicio» (González, 2001: 328).

Lo que caracteriza al proceso cognitivo del juicio de una persona moralmente responsable es la racionalidad, entendida desde la visión moral como la toma racional de decisiones, de forma no impulsiva, meditando las alternativas, consecuencias y riesgos, poniendo atención y procurando claridad en los propósitos, medios y fines (Goodpaster, 1983: 301), pero también está implicado el respeto, que involucra la especial consideración por las perspectivas de otros individuos que se vean afectados, y el tratamiento de las personas como fines y no como medios, en términos del imperativo categórico kantiano (Frankena en Goodpaster y Mathews, 1982: 4). Estos dos elementos, racionalidad y respeto, inciden en la manera en la que las personas deciden, porque afectan el momento

${ }^{8}$ Esta concepción está unida usualmente a contextos legales y morales, relacionada con la búsqueda de las causas de alguna acción o evento, para averiguar quién o quiénes son los responsables, ya sea para culparlos o para premiarlos por su actuación, subrayando especialmente el factor de causalidad en cuanto a las consecuencias producidas por los propios actos (Goodpaster y Mathews, 1982: 3).

${ }^{9}$ En esta acepción considera las normas sociales en los ámbitos en los que los individuos se ven sometidos por estas reglas, que son impuestas y que habitualmente están relacionadas con el rol de los sujetos en la sociedad (Goodpaster y Mathews, 1982: 3). 
en el que el individuo procesa la información y elige una opción de actuación. ${ }^{10}$ Pero ¿qué importancia tienen estos conceptos morales para la corporación? K. Goodpaster y J. Mathews opinan que la empresa pueda producir sus propios juicios y comportamientos morales, así como las personas actúan responsablemente reuniendo información sobre el impacto de sus acciones en otros y la usan para decidir, las organizaciones pueden hacer exactamente lo mismo. Esos dos componentes de la responsabilidad -la racionalidad y respeto- están bien definidos en estructura de decisiones internas de Peter French y son lo suficientemente precisos para ayudar a desarrollarla (Goodpaster y Mathews, 1982: 4).

Puede que los individuos responsables moralmente no estén siempre de acuerdo en las mismas normas éticas, pero al menos tienen la posibilidad de dialogar sobre ellas. Las empresas cuentan con la EDI para reflexionar sobre las reglas que definen las relaciones de autoridad entre los empleados y las políticas corporativas, logrando las condiciones de un ambiente que les permita decidir responsable y moralmente (González, 2001: 329-331).

A través del estudio de la responsabilidad moral individual, se puede ver fácilmente las diferencias de responsabilidad moral entre corporaciones, de la misma manera que se observa entre diferentes individuos. Algunas empresas establecen en sus políticas, sistemas de incentivos, organigramas, sistemas de control interno, agendas de investigación estratégica. Todos estos elementos podrían denominarse autocontrol, integridad $y / o$ conciencia en una persona. Otras instituciones están preocupadas por sus consumidores, empleados y el resto del público en modos que otras claramente no lo están, preocupándose sólo por los beneficios económicos que pueden producir a costa del ambiente, trabajadores y clientes (Goodpaster y Mathews, 1982: 4-5).

Como las personas, las corporaciones pueden revelar su carácter por medio de las políticas que implantan y el impacto que pueden tener sobre la gente, rechazando procesos de producción ${ }^{11}$ o normas que sean cuestionables moralmente. El concepto de responsabilidad moral no solo tiene sentido si es aplicado en las organizaciones, sino que además proporciona claves para diseñar modelos de políticas corporativas más efectivos que los existentes (Goodpaster y Mathews, 1982: 4-6).

\footnotetext{
10 Por ejemplo, una persona racional, pero no respetuosa no mentiría, a menos que sea razonablemente seguro hacerlo porque no lo descubrirán. Un sujeto que decide respetuosa y racionalmente se interesa por si las consecuencias de su comportamiento suponen daños a otros (Goodpaster y Mathews, 1982: 4)

${ }^{11}$ Por ejemplo, los efectos que causan los alimentos transgénicos o el uso de combustibles fósiles.
} 


\section{Adela Cortina}

Cuando una empresa se crea no posee una "primera naturaleza» como dirían los clásicos, sino solamente cuentan con la misión y visión del proyecto, al igual que con los caracteres de los emprendedores que inician su negocio. Pero con el paso del tiempo, la empresa se constituye como una real organización, que se va haciendo cargo de sí a través de las decisiones que toma, determinaciones que no son hechas ni pueden ser imputadas a los dueños o empleados, porque fueron concebidas por un agente moral colectivo (Cortina, 1997: 18).

Las empresas tienen un tipo de moralidad análoga a las de las personas. Desde la tradición zubiriana estructurista se pueden identificar los rasgos que caracterizan a la moral como estructura en los humanos, y en ese sentido colaborar con la explicitación de la correspondencia de dichos rasgos a las empresas.

Los humanos como organismos inteligentes se ven obligados a adoptar una serie de comportamientos para lograr su superviviencia. No perciben el ambiente como un conjunto de estímulos, sino que lo inteligen como una realidad de la que deben hacerse cargo, porque su estructura así lo exige además de vegetar y sentir. Justamente de esa necesidad de su sistema animal, de hiperformalizarse, surge el primer momento de libertad, en un sentido básico y amplio. Para hacerse cargo de su realidad no abandona los estímulos que se le presentan sino que los aprehende, analizando las distintas vías de acción, "adaptándose» al medio y haciendo que el medio de "adapte» a sus necesidades. Así, toma una decisión, elige una forma de actuación, de la que es responsable, y lo hace de forma consciente. El organismo humano se apropia de las posibilidades que va eligiendo convirtiendo lo querido en sido, para adueñarse de sí mismo. Ese carácter configura la identidad, una especie de definición de la persona, cuestión que debe hacer durante toda su vida. Identidad que posee tres dimensiones diferentes, la moral, la personal y la social (Cortina, 1998: 126-128; Zubiri, 1992: 74-76; 1996: 43102).

Las empresas tienen una estructura organizada semejante a la estructura constitutiva de un organismo humano, a su sustantividad. Razón por la cual se pueden identificar rasgos de ésta en aquella y argumentar que las empresas si son agentes morales. A partir de la analogía, Adela Cortina establece que la estructura moral de las organizaciones, está sustentada por tres aspectos, la responsabilidad por las decisiones, la conciencia corporativa y la identidad empresarial.

En el procedimiento a través del cual se toman las decisiones empresariales no participan unos cuantos individuos -como suele pensarse, tal vez solo los accionistas o directores-, sino que se realiza por 
medio de una especie de red en la que se comparte información, ${ }^{12}$ se delibera y se decide un rumbo de actuación, siendo responsable la organización como un ente estructurado y no de forma individual cada uno de sus miembros. Así como según al tradición zubiriana, los organismos por sus notas constitutivas son capaces de captar el ambiente, captar los estímulos que se le presenten, comprendiéndolos y respondiendo desde ese primer momento de libertad, procurando que la realidad física concuerde con la realidad querida, emprendiendo cursos de acción determinados, y justificando su decisión, las empresas como organizaciones estructuradas, también pueden ser responsables, no solo de sus decisiones sino de las consecuencias de ellas (Cortina, 1998: 128129; Zubiri, 1986: 93-100).

Las empresas también poseen conciencia, en su modo particular de ser. No es una conciencia exactamente igual a la de una persona, pero se puede decir que es equivalente, ya que pueden sopesar sus acciones, ponderar y valorar si están persiguiendo sus objetivos de manera apropiada, reflexión "grupal» que requiere que todos los miembros conozcan los objetivos y metas que persiguen, además de haber expresado su intención de cooperar o actuar en pro de ellos. Este comportamiento permite que la empresa se apropie de sus decisiones, que no las deje al azar o en manos de otros. Es como un proceso institucional que dota a la empresa con un carácter autonómico, el cual tiene cinco operaciones claves: articular, educar, escuchar, reflexionar y revisar los valores adoptados con vistas a la toma de decisiones. Además, dicho momento es muy importante para la relación de la misma con los stakeholders y con los afectados de las actividades empresariales. En este sentido, el balance social también se convierte en una herramienta para la gestión de la organización (Cortina, 1998: 129-131).

El otro aspecto que compone la estructura moral de las empresas, junto con la responsabilidad y la conciencia corporativa, es la identidad. La cultura organizacional entendida como el conjunto de valores, creencias e ideales compartidos, puede ser orientada de diferentes maneras, por ejemplo maximizar beneficios a toda costa o mantener la viabilidad y competitividad de la organización éticamente. Esa cultura que va formando el êthos, se convierte en identidad, distendida en tres niveles. La identidad moral, como indica A. Cortina, «define el horizonte moral en el que la organización toma decisiones al conceder un mayor valor a una actuaciones que a otras», la identidad organizativa "muestra su originalidad su carácter específico frente a otras organizaciones», y la identidad social «precisa el reconocimiento del público para identificarse a sí misma» (1998: 131).

\footnotetext{
12 Según el tipo de empresa y la cultura organizacional que posea, esa "red" será más abierta y horizontal, o vertical y cerrada, dándole a sus miembros mayor o menor libertad para participar en las decisiones empresariales.
} 


\section{Réplicas individualistas}

\section{Nani Ranken}

La analogía entre personas y corporaciones de K. Goodpaster y J. Mathews que cimenta la agencia moral corporativa se debilita en el momento en el que se busca un motivo para el desarrollo moral, así lo expresa Nani Ranken, quien considera que los miembros de una corporación son los verdaderos agentes morales, por lo que la actividad corporativa debe estar basada en el reconocimiento de estos individuos (1987: 633).

Esta autora no critica ni el análisis de la responsabilidad moral ni las implicaciones que K. Goodpaster y J. Mathews extraen para la toma de decisiones empresariales, lo que le parece ineficiente es la analogía en la que se le atribuyen aspectos individuales humanos a las corporaciones para hacerlas responsables. La proyección moral se muestra claramente insuficiente ante la pregunta de cómo puede una empresa puede ser un ente moralmente responsable -debe reunir y procesar información, tomar en cuenta el impacto moral en otro, debe razonar e incluir consideraciones morales en sus razonamientos, debe coordinar intereses que se vean en conflicto, combinar medios con fines y hacer lo que sea necesario para implementar su decisión. Son demasiados procesos y de inviable ejecución para ser llevados a cabo en cada una de las decisiones que la tome compañía (Ranken, 1987: 633).

Desarrollar hábitos es algo que a las personas puede resultarles motivador, al contrario que para las empresas. La institucionalización de características morales no es un elemento que motive a una corporación. Éstas no poseen ninguna fibra interna que las motive como a los humanos. Son las personas, desde adentro o desde afuera de la corporación, quienes deciden cómo institucionalizar los elementos que corresponden a la responsabilidad moral y por ser un proceso implementado por personas que ocupan cargos importantes, es de ellas de dónde debe provenir el sentido moral. Las empresas no pueden ejecutar esta tarea por si solas, son instituciones, no pueden institucionalizar a sí mismas (Ranken, 1987: 634).

Para N. Ranken el análisis de K. Goodpaster y J. Mathews no presenta ningún descubrimiento importante, sino que es una mala adjudicación del principio. Es un proceso que es aplicado a personas, pero que en el principio de proyección moral es empleado en las organizaciones, en el cual aconsejan perfectamente a los gerentes cómo deben hacer para institucionalizar la ética en términos estructurales, pero no responde a la pregunta sobre qué hacer para motivar a la gente que trabaja en la empresa para que implementen esa institucionalización (Ranken, 1987: 635). 
La corporación no debe servirse ni protegerse por su propio bien, ${ }^{13}$ es una simple herramienta humana que no tiene valor intrínseco, a diferencia de las personas, que sí tienen un valor característico, el de los seres vivos, por su capacidad de supervivencia y su racionalidad (Ranken, 1987: 637). Para esta autora la analogía de la persona-corporación no es fructífera, no ayuda a motivar a la personas a desarrollar hábitos responsables en el desempeño de sus roles organizacionales, sino todo lo contrario.

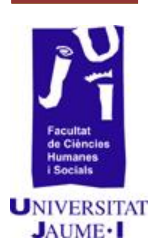

\section{Milton Friedman}

El economista ganador del premio Nobel, Milton Friedman, intenta delimitar el ámbito de la responsabilidad social empresarial (RSE) y para ello se pregunta primero quién es el responsable y a quién se le puede imputar responsabilidades (González, 2001: 314).

Los discursos sobre la responsabilidad social de las empresas y sus negocios se caracterizan -en su opinión- por su imprecisión analítica y su falta de rigor. ¿Qué significa afirmar que las empresas tienen responsabilidades? Tan sólo la gente tiene responsabilidades. Una corporación es una entidad artificial y en este sentido puede tener responsabilidades artificiales, pero no se puede afirmar que las empresas tengan responsabilidades por sí mismas, ni siquiera en sentido abstracto (Friedman, 1970: 1). La postura de M. Friedman es que únicamente de modo individual y personal se puede ser responsable, porque no se pueden adscribir responsabilidades a las empresas o instituciones.

El autor se centra en los altos ejecutivos como sujetos de responsabilidad, debido a que son éstos quienes tienen la obligación de dirigir las empresas guiándose por los intereses que muestran los dueños de la organización o los accionistas que tienen el control, ya que son sus empleadores (Friedman en González, 2001: 314-315).

La empresa como tal no posee una responsabilidad social corporativa propia. Los directivos no tienen responsabilidades sociales específicas simplemente por desempeñar su cargo. Su única responsabilidad es de carácter económico, la de generar las mayores ganancias posibles, ya que para eso fueron contratados (Friedman, 1970: 1). La responsabilidad social sólo les incumbe a los empleados de las instituciones públicas, quienes fueron elegidos democráticamente para ello (Friedman en

\footnotetext{
${ }^{13}$ Cuando los empleados se ven a ellos mismos sirviendo a la empresa, en un sentido donde la empresa es percibida como una superpersona con vida propia e intereses, entonces algunos asuntos morales serán descartados por los que sean más relevantes. Por ejemplo, en los hospitales trabajan doctores y enfermeras, y ellos están al servicio de los pacientes, ese es su rol. Sería muy desafortunado que los empleados vieran al hospital como a la persona que deben servir, porque desestimarían a los pacientes que justamente son las personas con las que tienen la verdadera responsabilidad. La condición ideal es que los trabajadores se vean comprometidos libremente en su labor, y que puedan naturalmente hacer uso de las categorías morales mientras que se informan, evalúan y deciden qué alternativas tomar en el curso de su desempeño laboral (Ranken, 1987: 637).
} 
Achbar, Abbott y Bakan, 2003). M. Friedman diferencia así los objetivos empresariales de los sociales. La responsabilidad social debe ser una preocupación individual de cada ciudadano y de los líderes que elija la sociedad para desarrollarla (González, 2001: 316).

\section{John Ladd}

A diferencia de la postura que asume M. Friedman, John Ladd se apoya en la separación entre moral y empresa, tratando de explicar la desconexión entre ética y economía, la disociación entre objetivos morales, sociales, empresariales y económicos (González, 2001: 317).

Las empresas están controladas por sus estructuras. Por tanto, según J. Ladd, están incapacitadas para ejercer la libertad moral. No pueden ser sujetos de responsabilidad pues los individuos son los que actúan por ellas, sólo los miembros de las organizaciones pueden tener responsabilidades. La empresa no puede ser culpada por sus acciones, porque éstas son productos de su estructura (Donaldson, 1982: 23). Las corporaciones no pueden ser definidas como agentes morales porque son parte de una clasificación muy especial que las incapacita para llegar a tener motivos morales. Son parte de las organizaciones formales, ${ }^{14}$ como también lo es una burocracia gubernamental.

Cuando se dice que las empresas actúan racionalmente, lo que se afirma es que actúan de acuerdo a políticas organizacionales respetadas por sus miembros, pero esto no significa que la entidad deba definirse como un agente moral, lo que se contrasta con que los conceptos éticos

no pueden ser aplicados de modo directo a las corporaciones como si éstas fueran personas; esto no quiere decir que no puedan ser aplicados de modo indirecto [...] a los individuos o a los grupos de individuos asociados con las corporaciones de algún modo u orden (Ladd en González, 2001: 318).

Las nociones morales no pueden ser aplicadas directamente a las organizaciones formales porque el marco teórico lógico de la estructura de toma de decisiones empresarial excluye el tipo de razonamiento moral que se exige a los individuos. "Cuando las organizaciones actúan, son los seres humanos quienes actúan» (Ladd en González, 2001: 319). Para Ladd, las corporaciones no son personas, sino organizaciones de personas.

\section{John Danley}

Para lograr invalidar la tesis de Peter French, el filósofo John Danley argumenta que para atribuir responsabilidades existen al menos seis

\footnotetext{
${ }^{14}$ Las organizaciones formales son aquellas unidades planeadas, deliberadamente estructuradas para el propósito de atender metas específicas (Donaldson, 1982: 23)
} 
maneras diferentes de adscribirla según la clasificación de Feinberg (1970), de las cuales P. French solamente toma en cuenta tres, pero los otros tres tipos de adscripciones son importantes porque reflejan parte de la lógica de la constitución de un agente responsable (Danley, 2002: 244). P. French falla -según Danley- en tratar de demostrar a través de su análisis de la atribución de responsabilidad. Que una empresa haya hecho algo no quiere decir que sea culpable o que su actuación implique alguna obligación. ${ }^{15}$

La realidad organizacional no es la que está detrás de la tesis de P. French debido a que existen algunas decisiones que no se apegan a la EDI ni al organigrama, decisiones que se llevan a cabo y que tienen consecuencias, que son respetadas y aceptadas por los miembros de la organización, pero según esta teoría colectivista serían hechos ilegales y no tendrían reconocimiento (Danley, 2002: 243-245). Esto implica que la organización nunca podría actuar ilegalmente. Aunque los directores votaran unánimemente para involucrar a la empresa en un acto ilegal, esto no podría denominarse como un acto de la empresa y -según Danleyefectivamente lo es, en caso de producirse tal proceso.

El sentido de responsabilidad se adscribe cuando los acontecimientos son causados intencionalmente por el agente, pero según la línea de Danley las empresas no poseen intenciones como las de una persona biológica. Tampoco cuerpo ni alma para ser condenados por los hechos que realizan. Sólo los individuos pueden sentir culpa y ser castigados. La adscripción de responsabilidades a las corporaciones y la pretensión de que éstas son agentes morales perturba la lógica del discurso moral, reduciendo a los sujetos morales humanos a ciudadanos de segunda clase en la expansión de la comunidad moral. ${ }^{16}$

\section{Mathew Altman}

Según la visión de Mathew Altman, no existen intenciones colectivas aparte de las intenciones de los agentes individuales, quienes actúan como miembros de la corporación, porque una organización en sí misma no tiene obligaciones morales.

En este sentido, M. Altman realiza una crítica a los filósofos que aplican la ética kantiana a las organizaciones y que usualmente utilizan la teoría de

\footnotetext{
${ }^{15}$ «Jones es responsable por soltar la pelota» no es más que decir que «Jones soltó la pelota» sin culpar o atribuir la culpa al agente (Danley, 2002: 243).

${ }^{16}$ Si una empresa es un agente moral ¿cuál es el estatus de sus empleados? ¿ison menos agentes morales que la corporación en la que trabajan? Un empleado podría ser un agente moral de grado 1 , un comité organizacional de toma de decisiones sería un agente moral de grado 2 , así sucesivamente, y entonces la empresa completa sería una agente moral de grado "n». Una empresa podría permitirse hacer lo que sea mientras no interfiera en la libertad de las otras empresas, las cuales también tendrían un grado «n»de agencia. Según este razonamiento las corporaciones podrían interferir en la libertad de los agentes individuales sin ningún tipo de violación moral, porque son agentes de grado «n» (Freeman y Werhane, 2009: 520).
} 
una manera que es contradictoria con sus propios requisitos metaéticos. M. Altman reconoce que es difícil relacionar el imperativo categórico con la corporación, pues el primero se sustenta en una concepción particular de la agencia moral que excluye la responsabilidad colectiva y, más específicamente, la responsabilidad corporativa (2007: 253).

Caracterizar a las empresas como agentes morales sería concebirlas incorrectamente y una transgresión de los límites de la teoría moral kantiana. En este sentido sólo se puede juzgar a los ejecutivos o empleados, pero no a la empresa. Los individuos no se encuentran restringidos simplemente por las normas morales porque pueden actuar, sino porque pueden elegir si un conjunto de máximas son acordes con la razón universal, cosa que la empresa no puede hacer. No existe una máxima organizacional distinta de las máximas de los individuos que conforman las corporaciones (Altman, 2007: 256).

Una empresa puede actuar, pero dada su falta de inclinaciones y su falta de razón, la organización no sería el tipo de ente que actúe motivado por deseos o por respeto moral. Según Kant, lo que cuenta moralmente es el por qué un agente realizó una acción. Bajo esta concepción, cualquier estrategia corporativa expone simplemente los objetivos de los empleados (Altman, 2007: 257).

Según M. Altman, las organizaciones no tienen conciencia moral que las limite. Por ello, no poseen humanidad en el sentido kantiano. Los miembros de una compañía pueden razonar sobre la base de las políticas organizacionales, pero esas políticas no son impuestas por la propia compañía, sino por sus directores y accionistas. No se puede atribuir agencia moral a las corporaciones sin antropomorfizarlas ilegítimamente (2007, 257-260).

\section{Propuesta de un concepto integrador}

Después de haber examinado las distintas propuestas a favor de la agencia moral corporativa, y sus variadas replicas, creemos pertinente la creación de un concepto integrador que englobe las características más importantes y compatibles de las tesis que expresan cómo las empresas son agentes que también se encuentran en la comunidad moral. Para procurar así, una base más sólida y compacta de su argumentación teórica, reduciendo sus resquicios, no solo por llevar a cabo una formalidad académica en la fundamentación filosófica del concepto, sino por la importancia que conlleva aceptar y reconocer que las empresas si son agentes, responsables moralmente de su comportamiento y las posibles consecuencias de los mismos.

La propuesta ha sido concretada en la siguiente figura, que será explicada en párrafos sucesivos. 


\section{Libertad}

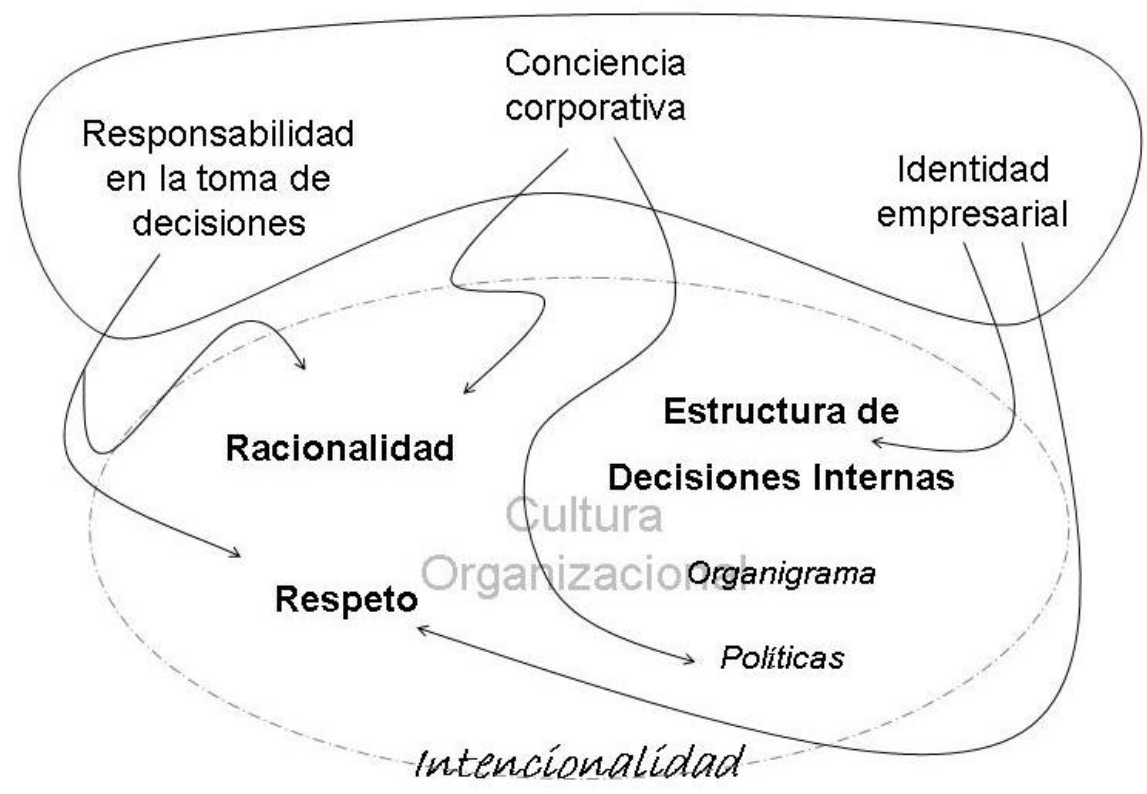

Figura 1. Propuesta integradora de agencia moral corporativa ${ }^{17}$

Partiendo del primer momento de libertad que tiene la empresa, ésta debe hacerse cargo de sí como un organismo inteligente que se encuentra con la realidad; y no de cualquier manera, sino que debe adueñarse de sí misma, eligiendo concienzudamente de entre las posibilidades que se le presentan, respondiendo por las opciones de actuación que ha descartado y respondiendo también por las consecuencias que produzca el rumbo de actuación que decidió tomar. Así, puede ir construyéndose por y para sí misma. Como Adela Cortina nos indica, este proceso repetitivo que realiza la empresa, el de apropiación de la realidad, está constituido por de tres notas formales que se pueden y se deberían dar simultáneamente, la conciencia corporativa, la responsabilidad por la toma de decisiones y la identidad empresarial. Esto es la estructura moral de las organizaciones.

Esas notas formales son expresadas por medio de una trama de conceptos que permiten facticamente que las empresas, como sujetos de acción, se adueñen de sí mismas, y se conviertan en unos agentes morales colectivos. La racionalidad y el respeto que Goodpaster y Mathews desarrollaron en su propuesta sobre la agencia moral corporativa, junto con la noción de la Estructura de Decisiones Internas (EDI) de Peter French, conforman los elementos organizacionales que constituyen la malla de conocimiento interactivo, que denominamos cultura organizacional. También es importante destacar que existe una característica común en cada una de las notas formales de la estructura

\footnotetext{
${ }^{17}$ Gráfico de elaboración propia, construido a partir de las propuestas de A. Cortina, P. French, K. Goodpaster y J. Mathews.
} 
moral de las organizaciones, que es la intencionalidad, así como en los conceptos que la expresan facticamente. Cada uno de estos aspectos se desarrollan, siempre, a partir de una intención colectiva, es decir, en parte esos aspectos son posibles gracias al entramado de fondo de la cultura de la empresa.

La responsabilidad en la toma de decisiones se expresa a través de racionalidad que posee la organización en cuanto a la manera en la que actúa, de modo analítico, racional, sopesando las alternativas que tienen, las posibles consecuencias y los riesgos, tratando de armonizar/adaptar la realidad con los objetivos organizacionales. Al mismo tiempo, esta nota de la estructura moral de las empresas también se manifiesta a través del respeto, como una concepción en la que la organización considera a los stakeholders y a los afectados legítimos en sus procesos deliberativos y productivos siempre como fines y nunca como medios.

La conciencia corporativa se muestra por medio de los conceptos de racionalidad y de la Estructura de Decisiones Internas, particularmente en las Políticas de la empresa. En cuanto al primer aspecto, las empresas lo demuestran por su capacidad ponderar, evaluar, valorar si están persiguiendo los objetivos apropiadamente, es decir, aptitud reflexiva deliberada. El segundo, las Políticas, se manifiesta como conciencia empresarial en sus creencias y valores, y en sus reglas de reconocimiento que facilitan la toma colectiva de decisiones en diferentes niveles organizacionales.

La identidad de la empresa se evidencia en la Estructura de Decisiones Internas, en todas sus partes, tanto en el organigrama como en las políticas. Son la misión, visión, valores, estructura de responsabilidades de cargos y funciones, de relaciones inter y de-pendientes, al igual que todas las reglas de reconocimiento, que se constituye el carácter de la empresa. Así como también en la noción de respeto, al tomar en cuenta a los grupos de interés y a los afectados en el proceso de definición y progresiva construcción de su êthos corporativo.

\section{Conclusiones}

Los sujetos son capaces de elegir cómo actuar, de manera responsable a través de acciones intencionales, asumiendo los aspectos incidentales concienciados y los posibles efectos secundarios de esas acciones. La moralidad del agente sobre sus intenciones yace, no en el contenido de éstas, sino en el ejercicio de su autonomía como sujeto responsable, en su capacidad de evaluación, apropiación y reflexión de las intenciones que posea. Ahora bien ¿cómo se realiza esa evaluación, apropiación y reflexión de las intenciones y posterior acción en las empresas? Para comprender este fenómeno, realizamos la revisión de las teorías clásicas sobre la agencia moral corporativa, así como algunas de 
sus réplicas, encontrando muchos puntos útiles y comunes en las primeras, al igual que críticas válidas e importantes en las segundas. Dicha situación nos motivó a elaborar un concepto integrador de agencia moral corporativa, fundamentado en la estructura moral de las empresas de Adela Cortina. Para desarrollar fácticamente tal estructura, contamos con las nociones de Racionalidad y Respeto de Goodpaster y Mathews, y con la Estructura de Decisiones Internas de Peter French.

La propuesta integradora que se presentó en este trabajo, procuró argumentar que las empresas son agente morales, con el fin de imputarles responsabilidad por sus actos y por todas aquellas consecuencias de las que resulten afectados grupos o individuos. Esta concepción moral de actuación colectiva es una de las bases para la construcción del concepto de engagement ético, entendido como vínculo moral entre la empresa y sus stakeholders, donde se intersectan intersubjetivamente los intereses de las partes. Dicha cuestión se intentará profundizar en investigaciones sucesivas.

A pesar de que en última instancia, las empresas están constituidas y manejadas por personas, su forma de actuación es análoga a la de sus creadores y desarrolladores aunque sean entes diferentes. Puede que en algunos casos la responsabilidad deba ser atribuida a individuos específicos, pero la mayoría de las acciones que realizan las corporaciones son colectivas, de no ser así, no estarían representando a la empresa como una entidad diferente a la de sus miembros. En palabras de Adela Cortina:

Cualquier entidad cuyas decisiones tengan consecuencias sociales debe asumir su responsabilidad social y pública por ellas, cosa que ocurre claramente en el caso de las empresas, cuyas decisiones tienen inevitablemente repercusiones sociales, y por tanto son responsables de ellas ante la sociedad a la que afectan (1997: 25).

\section{Bibliografía}

ACHBAR, M., ABBOTT, J. y BAKAN, J. (2003): The Corporations: Institutions or Psicopaths, escrita por Joel Bakan, producida por Mark Abbot, Maureen Levitt y Bart Simpson, Canada.

ALTMAN, M. (2007): "The Decomposition of the Corporate Body: What Kant Cannot Contribute to Business Ethics», Journal of Business Ethics, Vol. 74, $\mathrm{N}^{\circ} 3$.

CORTINA, A. (dir.) (1997): Rentabilidad de la ética para la empresa, Fundación Argentaria, Madrid.

- (1998): Hasta un pueblo de demonios, Ética pública y sociedad, Taurus, Madrid. 
DANLEY, J. (2002): "Corporate Moral Agency», Frederick, R. (ed.): $A$ Companion to Business Ethics, Blackwell Publishing, Oxford.

DAVIDSON, D. (2001): Essays on Actions \& Events, Clarendon Press, Oxford.

DONALDSON, T. (1982): Corporations and Morality, Prentice Hall College Div, Nueva York.

FEINBERG, J. (1970): Doing and Deserving, Princeton University Press, New Jersey.

FREEMAN, E. Y WERHANE, P. (2009): "Corporate Responsibility», BEAUCHAMP, T. y BRENKERT, G. (eds.): The Oxford Handbook of Business Ethics, Oxford University Press, Nueva York.

FRENCH, P. (1979): "The corporation as a moral person», American Philosophical Quarterly, Vol. 16, No. 3.

FREUND, E. (2000): The legal nature of corporations, Batoche Books, Kitchener.

FRIEDMAN, M. (1970): "The Social Responsibility of Business is to Increase its Profits», The New York Times Magazine.

GONZÁLEZ, E. (2001): "La responsabilidad moral de la empresa: una revisión de la teoría de stakeholder desde la ética discursiva», Tesis Doctoral, Universitat Jaume I, Castellón.

GOODPASTER, K. (1983): "The Concept of Corporate Responsibility», Journal Business of Ethics, Vol. 2, No. 1.

GOODPASTER, K. y MATHEWS, J. (1982): «Can a corporation have a conscience?», Harvard Business School Reprint.

HESSEN, R. (1979): In defense of corporation, Hoover Institution, Stanford.

MARENBON, J. (2003): Boethuis: Great Medieval Thinkers, Oxford University Press, Nueva York.

PETTET, B. (2005): Company Law, Pearson Education Limited, Harlow.

RANKEN, N. (1987): «Corporations as Persons: Objections to Goodpaster's "Principle of Moral Projection"», Journal Business of Ethics, Vol. 6, Nº 8.

ZUBIRI, X. (1986): Sobre el hombre, Alianza Editorial, Madrid.

- (1992): Sobre el sentimiento y la volición, Alianza Editorial, Madrid. 